\title{
Multiple Myeloma-Like Spinal MRI Findings in Skeletal Fluorosis: An Unusual Presentation of Fluoride Toxicity in Human
}

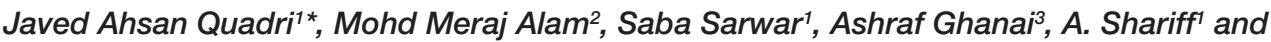 \\ Taposh K. Das'
}

'Anatomy, All India Institute of Medical Sciences (AIIMS), New Delhi, India, ${ }^{2}$ Surgery, Guwahati Medical College and Hospital, Guwahati, India, ${ }^{3}$ Endocrinology, Metabolism and Diabetes, All India Institute of Medical Sciences (AlIMS), New Delhi, India

Endemic fluorosis is a worldwide environmental problem due to excessive fluoride, commonly due to increased drinking water fluoride levels but sometimes due to other sources such as food with high fluoride content. In India, 21 of the 35 states are known to have health problems associated with fluoride toxicity. The present report is a case of a 50-year-old female who was seen with progressive spinal complications and a MRI of the spine suggestive of multiple myeloma. The MRI of the lumbosacral spine showed

OPEN ACCESS

Edited by:

Medhat M. Osman,

Saint Louis University, USA

Reviewed by:

Nghi Co Nguyen,

University of Pittsburgh, USA

Mustafa Erkan Altinyay, Acıbadem Bodrum Hospital,

Saudi Arabia

*Correspondence: Javed Ahsan Quadri javedaiims@gmail.com

Specialty section: This article was submitted to Cancer Imaging and Diagnosis,

a section of the journal

Frontiers in Oncology

Received: 14 August 2016 Accepted: 04 November 2016 Published: 21 November 2016

Citation:

Quadri JA, Alam MM, Sarwar S, Ghanai A, Shariff $A$ and Das TK (2016) Multiple Myeloma-Like Spinal MRI Findings in Skeletal Fluorosis: An

Unusual Presentation of Fluoride Toxicity in Human.

Front. Oncol. 6:245. doi: 10.3389/fonc.2016.00245 a diffuse and heterogeneous marrow signal of the lower dorsal and lumbosacral vertebrae. The MRI was also suggestive of coarse trabeculation and appeared predominantly hypointense on the T1W image and had mixed signal intensity on the T2W image. These findings were suggestive of neoplastic bone marrow infiltration and the presence of a proliferative disorder, with multiple myeloma being the most likely. During the patient workup, it was found that other family members were also having similar complications and, after investigation of these family members, it was found that they are suffering from systemic fluorosis. The patient was then evaluated for skeletal fluorosis, and this condition was found to be present. Multiple myeloma was ruled out by the finding of a negative serum protein electrophoresis. The spinal complications appeared to be mainly due to the compression of the spinal cord and nerve roots by protruding osteophytes, thickening of the posterior longitudinal ligament, and thickening of the ligamentum flavum resulting in a compressive myeloradiculopathy and compressive myelopathy. The finding of multiple myeloma-like findings on the spinal MRI in association with skeletal fluorosis was considered to be a very rare event. This case report underlines the need to consider the presence of spinal skeletal fluorosis when evaluating spinal complications with unusual pseudo-multiple myeloma-like changes on the spinal MRI.

Keywords: dual-energy X-ray absorptiometry, fluorotic myelopathy, MRI, M-protein electrophoresis, neoplastic bone marrow infiltration, spinal fluorosis

\section{INTRODUCTION}

Endemic skeletal fluorosis is widely prevalent in India, China, and in many other countries around the world (1). The primary findings of fluorosis are mottling of teeth, osteosclerosis, soft tissue calcification, and marginal bony overgrowth (2). Symptoms range from mild motor and sensory loss to spastic paraplegia and spastic quadriplegia with bladder and bowel incontinence. 
Association of fluoride toxicity with dysfunction of bone, and endocrine, gastrointestinal, and reproductive systems have been previously reported (3-5). Effects of fluoride on corticosteroids metabolism and its role in bone mineralization have also been reported by Das et al. In this case report, we are reporting very rare multiple myeloma-like MRI findings in a skeletal fluorosis patient.

\section{CASE REPORT AND DESCRIPTION}

We are presenting a case report of a 50-year-old female from a village of Agra, in the state of Uttar Pradesh, an endemic fluorosis region in India. Patients belong to lower socioeconomic strata, and the source of income is agriculture. Patient was referred from district hospital to the supper specialty cancer hospital for the treatment of multiple myeloma, on the basis of radiological (MRI spine) findings. Patients represented with hemogram values as: hemoglobin $9.0 \mathrm{~g} / \mathrm{dl}$ (12.0-15.0), RBC $3.61 \times 10^{6} / \mu \mathrm{l}(4.0-4.9)$, HCT-28.2\% (36-44), MCV-78.1fL (80-100), MCH-24.9 g/dl (26-34), RDW-18.7\% (<14.5), and platelets count was normal $\left(268 \times 10^{3} / \mu \mathrm{l}\right)$, while ESR $40 \mathrm{~mm}$ (normal value for women over 50 years old: $<30 \mathrm{~mm} / \mathrm{h}$ ) was significantly raised after $1 \mathrm{~h}$, alkaline phosphatase was increased to $733 \mathrm{IU} / 1$ (33-131 IU/1). Lymphoma was ruled out, because lymphoma patients usually present with symptoms such as lymphadenopathy, night sweats, weight loss, dyspnea, itching, etc. All these symptoms were absent in the patient accept mild to moderate weight loss. The chances of renal osteodystrophy were negligible because osteodystrophy is a bone disease that occurs when patient's kidneys fail to maintain proper levels of calcium and phosphorus in the blood. The blood calcium level in the subject was $9.1 \mathrm{mg} / \mathrm{dl}$ (normal range $8.1-10.4 \mathrm{mg} / \mathrm{dl}$ ), renal osteodystrophy was ruled out. Because the serum level of PTH was in normal $49.06 \mathrm{pg} / \mathrm{ml}$ (normal range 15-65 pg/ml), hyperparathyroidism was least likely.

The patient was advised for MRI spine to diagnose the cause of severe spinal pain and stiffness. MRI of the lumbosacral spine showed a diffusely and heterogeneous marrow signal of visualized lower dorsal and lumbosacral vertebrae, their posterior elements as well as pelvic bones. MRI was also suggestive of coarse trabeculation and appears predominantly hypointense on T1W and mixed signal intensity on T2W image. These findings were suggestive of neoplastic bone marrow infiltrative and proliferative disorders and are more in favor of multiple myeloma rather than metastasis and other disease (Figures 1A,B). The MRI findings such as appearance of normal ligamentum flavum with no hypertrophy and normal appearance of pre- and paraspinal soft tissue were not suggestive of skeletal fluorosis also.

\section{THE SEQUENCE OF MRI FINDINGS OF LUMBOSACRAL SPINE}

Lumbosacral spine was examined in the sagittal, coronal, and axial planes as per the standard protocol. Both T1- and T2-weighted images were obtained. Additionally, fat sat sagittal
T2 images were also obtained. Diffusely altered and heterogeneous marrow signals of visualized lower dorsal and lumbosacral vertebrae and their posterior elements as well as pelvic bones were observed. These show coarse trabeculation and appear predominantly hypointense on T1W and mixed signal intensity on T2W images. A remarkably reduced, L2 -5 vertebral body height, mid-body wedging, and biconcave disk margins were noted. Concentric disk bulge (wide-based posterior central) protrusion at L4-5 causing thecal sac compression, bilateral neural foraminal narrowing, and compression of bilateral traversing L5, exiting L4, and cauda equina nerve roots. The MRI findings were also suggestive of concentric disk bulge at L3-4 causing thecal sac indentation, bilateral neural foraminal narrowing and bilateral exiting of L 3 nerve roots. The disk bulge at L1-2 and L2-3 causing thecal sac indentation and bilateral neural foraminal narrowing observed. Central canal stenosis was seen from D11 to S1. Pre-thecal epidural fat was well maintained without any significant abnormality. Thecal sac shows normal appearance at rest of the levels. The conus medullaris and cauda equina also appeared normal. There is no focal area of cord expansion or cord edema seen. No intraspinal mass is seen. Neural foramina and exiting nerve roots appear normal at rest of the levels. Articular facet and facet joints are normal. No ligamentum flavum hypertrophy was identified. Pre- and paraspinal soft tissue appeared normal.

\begin{tabular}{lc}
\hline Saggital diameter of bony spinal canal & Size in $\mathbf{~ m m}$ \\
\hline D11-12 & 02 \\
D12 & 10 \\
D12-L1 & 05 \\
L1 & 10 \\
L1-L2 & 04 \\
L2 & 09 \\
L2-3 & 04 \\
L3 & 07 \\
L3-4 & 04 \\
L4 & 06 \\
L4-5 & 04 \\
L5 & 07 \\
L5-S1 & 04
\end{tabular}

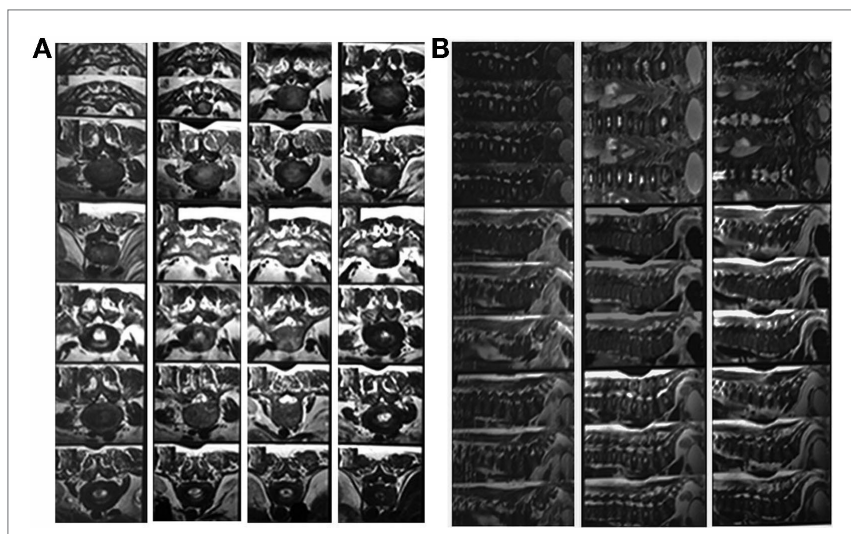

FIGURE 1 | (A,B) MRI findings showing multiple myeloma-like changes. 


\section{IMPRESSION OF MRI}

Diffusely altered and heterogeneous marrow signals of visualized lower dorsal and lumbosacral vertebrae, their posterior elements as well as pelvic bones were observed. This show coarse trabeculation and appears predominantly hypointense on T1W and mixed signal intensity on T2W images. These are suggestive of neoplastic marrow infiltrative/proliferative disorders and are more in favor of multiple myeloma rather than metastasis. Clinicopathological and biochemical correlations were advised.

\section{DIAGNOSIS AND CONFIRMATION OF SKELETAL FLUOROSIS}

Suspicion about the systemic fluorosis started during the patient workup when the patient reported that the other family members also suffered from the same kind of complications. After evaluation of family members, we found that they were having clear sign of dental fluorosis in children and skeletal fluorosis in adults and were symptomatic for fluorosis, which includes progressive pain and stiffness of neck, spine and limb joints, muscular weakness, lethargy, and gastrointestinal problems. After radiological and laboratory investigations, family members of the patients were diagnosed and confirmed for fluorosis. As per the family history, it was suspected that the radiological findings of multiple myeloma in the patients may be an unusual manifestation of chronic fluoride toxicity. To confirm the hypothesis, patient's urinary and serum fluoride concentration were measured by using selective fluoride ion electrode (Orion, Thermo Scientific), and it was found that fluoride level in urine and serum were significantly increased. To further confirm the chronic fluoride toxicity, forearm interosseous membrane calcification was evaluated by $\mathrm{X}$-ray and was positive for the same (Figure 2A), and X-ray hip was also suggestive of increased bone density (Figure 2B). DXA scan (bone densitometry) showed that the bone density increased manyfold, which is also suggestive of skeletal fluorosis (Figures 3A,B).

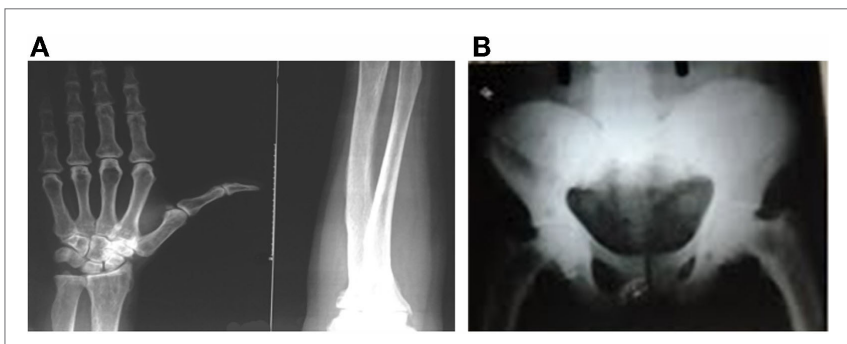

FIGURE 2 | X-ray, (A) forearm showing interosseous membrane calcification, (B) X-ray hip showing ectopic calcification.

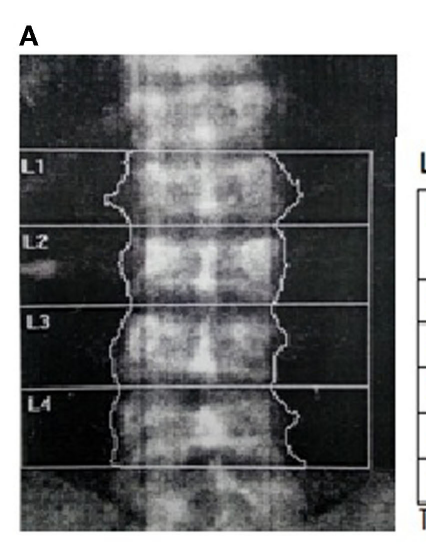

B

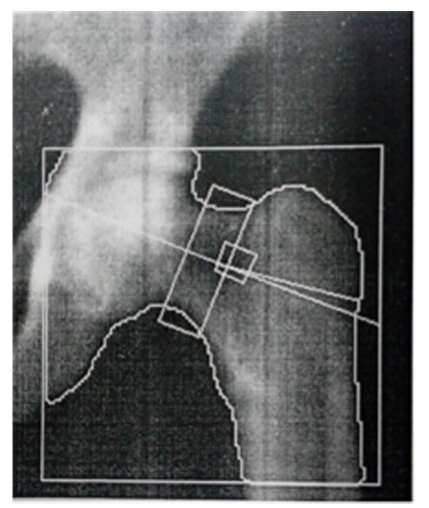

Lumbar spine DXA result Summary:

\begin{tabular}{|l|l|l|l|l|l|}
\hline Region & $\begin{array}{l}\text { Area } \\
\left(\mathrm{cm}^{2}\right)\end{array}$ & BMC $(\mathbf{g})$ & $\begin{array}{l}\text { BMD } \\
\left(\mathrm{g} / \mathrm{cm}^{2}\right)\end{array}$ & T-score & Z-score \\
\hline $\mathrm{L} 1$ & 15.09 & 24.27 & 1.608 & 5.6 & 6.3 \\
\hline $\mathrm{L} 2$ & 15.87 & 25.87 & 1.631 & 5.5 & 6.2 \\
\hline $\mathrm{L} 3$ & 16.98 & 28.15 & 1.657 & 5.2 & 6.0 \\
\hline $\mathrm{L} 4$ & 18.55 & 30.67 & 1.653 & 5.4 & 6.2 \\
\hline Total & 66.50 & 108.95 & 1.639 & $\mathbf{5 . 4}$ & 6.1 \\
\hline
\end{tabular}

Total BMDCV $1.0 \%$, ACF $=1.031, \mathrm{BCF}=0.999, \mathrm{TH}=7.361$

Left Hip DXA result Summary:

\begin{tabular}{|l|l|l|l|l|l|}
\hline Region & $\begin{array}{l}\text { Area } \\
\left(\mathbf{c m}^{2}\right)\end{array}$ & BMC $\mathbf{( g )}$ & $\begin{array}{l}\text { BMD } \\
\left(\mathbf{g} / \mathbf{c m}^{2}\right)\end{array}$ & T-score & Z-score \\
\hline Neck & 5.42 & 5.73 & 1.057 & 1.9 & 2.6 \\
\hline Troch & 11.36 & 9.63 & 0.848 & 1.4 & 1.9 \\
\hline Inter & 26.05 & 33.95 & 1.303 & 1.3 & 1.6 \\
\hline Total & $\mathbf{4 2 . 8 3}$ & 49.31 & 1.151 & 1.7 & $\mathbf{2 . 2}$ \\
\hline Ward's & 1.13 & 0.74 & 0.658 & -0.6 & 0.7 \\
\hline
\end{tabular}

Total BMD CV 1.0\%, ACF $=1.031, \mathrm{BCF}=0.999, \mathrm{TH}=7.361$ 
TABLE 1 | Fluoride levels in the serum, urine, and drinking water of the patient.

\begin{tabular}{lccc}
\hline Fluoride level in & Observed values (ppm) & Normal value (WHO) & Remarks \\
\hline Urine & 9.6 & Up to $0.10 \mathrm{ppm}$ & $\uparrow$ \\
Serum & 0.31 & Up to $0.02 \mathrm{ppm}$ & $\uparrow$ \\
Drinking water & 10.01 & Up to $1.00 \mathrm{ppm}$ & $\uparrow$ \\
\hline
\end{tabular}

The MRI findings were suggestive of multiple myeloma but other findings were indicating toward unusual outcome of chronic fluoride toxicity. The gold standard molecular marker for multiple myeloma is the presence of high molecular weight M-protein band in serum and urine, which clearly appears in gel electrophoresis. Therefore, to confirm and/or rule out the multiple myeloma, urinary and serum $\mathrm{M}$-protein electrophoresis was done. However, M-band was absent in urine and serum of the patient, thereby not confirming the diagnosis of multiple myeloma in contrast to MRI findings of multiple myeloma. Multiple myeloma was also ruled out by histopathological examinations. As per the findings such as high concentration of fluoride in urine, serum, and drinking water (Table 1), forearm interosseous membrane calcification, and increased bone density, it was established that this is an unusual manifestation of chronic fluoride toxicity.

\section{DISCUSSION}

Fluorosis in humans predominantly has dental and skeletal manifestation. In the early stage of chronic fluoride toxicity, patients are asymptomatic or gastrointestinal symptoms may be present. In the advanced stages, skeletal fluorosis causes crippling deformities and neurological complications. Neurological complications occur in $5-10 \%$ of cases of skeletal fluorosis (6). These features usually develop after exposure to a high fluoride content for longer period of time (7). Spinal cord involvement occurs in the cervical region followed by thoracic and lumbar region (8). These complications appear mainly due to the compression of the spinal cord and nerve roots by the protruding osteophytes, thickening of the posterior longitudinal ligament, and thickening of the ligamentum flavum resulting in compressive myeloradiculopathy and compressive myelopathy $(9,10)$, but multiple myeloma-like findings are very rare events and from the best of our knowledge, we are reporting this finding the first

\section{REFERENCES}

1. Susheela AK. Treatise on Fluorosis: 3rd Revised Edition. Delhi, India: Fluorosis Research \& Rural Development Foundation (2006).

2. Singh VP. Fluoride disorders: geological Linkages, human health and preventive measures. In Proceedings: Workshop on Medical Geology; 2004; Nagpur, India: IGCP-454, GSI Special Publication No. 83 (2004); p. 118-22.

3. Susheela AK, Das TK. Chronic fluoride toxicity: a scanning electron microscopic study of duodenal mucosa. J Toxicol ClinToxicol (1988) 26(7):467-76.

4. Das TK, Susheela AK. Effect of long-term administration of sodium fluoride on plasma calcium level in relation to intestinal absorption and urinary excretion in rabbits. Environ Res (1993) 62(1):14-8. doi:10.1006/enrs.1993.1084 time. In fluorosis, the compressive myelopathy has been observed to occur at different levels, but MRI findings suggesting multiple myeloma-like changes was a very unusual finding in fluorosis patient whom we have observed. Though the fluorosis was confirmed by the lab test and by other investigations such as high level of fluoride in body fluid, forearm interosseous membrane ossification/calcification, and increased bone density. M-band in serum and urine was negative, thereby ruling out MRI findings of multiple myeloma and suggesting that fluoride toxicity could probably be bringing out pseudo-multiple myeloma-like changes in the lumbosacral spine. These abovementioned MRI findings with systemic fluorosis are very rare events. Bone marrow aspiration and spinal marrow biopsy are invasive technique and cannot be advised routinely to rule out these types of pseudo-multiple myeloma-like MRI findings in spinal fluorosis. In such type of fluorosis patients if MRI spine is suggestive of multiple myeloma, the spinal biopsy will rule out multiple myeloma but will not suggest fluorosis and yet it is difficult to diagnose. Therefore, this case report will provide a dimension to think about rare spinal fluorosis that may show unusual multiple myeloma-like findings in spinal MRI.

\section{CONCLUSION}

The multiple myeloma-like MRI findings suggestive of neoplastic bone marrow infiltration and proliferative changes in the spine of fluorosis patients are very unusual, and outmost care should be taken in the diagnosis of such kind of fluoride toxicity. This case report contributes to the literature the possibility of finding multiple myeloma-like changes in the spine due to chronic fluoride toxicity. However, this observation needs to be further investigated in other fluorosis patients with spinal involvement to establish the fact that fluoride toxicity can bring about multiple myeloma-like changes in the spine.

\section{AUTHOR CONTRIBUTIONS}

Dr. JQ: patient workup and diagnosis. Dr. MA: interpretation of radiological finding and correlation with clinical findings. Ms. SS: fluoride estimation and diagnosis. Dr. AG: patient workup and idea generation about may be the case of fluorosis. Dr. AS: manuscript preparation. Dr. TD: cracked the case and confirmed the diagnosis (Dr. TD died due to his chronic illness).

5. Das TK, Susheela AK, Gupta IP, Dasarathy S, Tandon RK. Toxic effects of chronic fluoride ingestion on the upper gastrointestinal tract. J Clin Gastroenterol (1994) 18(3):194-9. doi:10.1097/00004836-199404000-00004

6. WHO. Fluorides (Vol. 227). Geneva: Environmental Health Criteria (2002). p. 14-6.

7. Reddy DR. Neurology of endemic skeletal fluorosis. Neurol India (2009) 57:7-12. doi:10.4103/0028-3886.48793

8. Reddy DR, Prasad VS, Reddy JJ. Neuroradiology of skeletal fluorosis. Clin Neurosci Ann Acad Singapore (1993) 22:493-500.

9. Haimanot RT. Neurological complications of endemic skeletal fluorosis, with special emphasis on radiculo-myelopathy. Paraplegia (1990) 28:244-51. doi:10.1038/sc.1990.31 
10. Naidu MR, Reddy DR, Reddy PK, SastryKolluri VR. Fluorotic cervical spinal cord disease. Rev Chir Orthop Reparatrice Appar Mot (1989) 75:228-31.

Conflict of Interest Statement: The authors declare that the research was conducted in the absence of any commercial or financial relationships that could be construed as a potential conflict of interest.
Copyright (c) 2016 Quadri, Alam, Sarwar, Ghanai, Shariff and Das. This is an open-access article distributed under the terms of the Creative Commons Attribution License (CC BY). The use, distribution or reproduction in other forums is permitted, provided the original author(s) or licensor are credited and that the original publication in this journal is cited, in accordance with accepted academic practice. No use, distribution or reproduction is permitted which does not comply with these terms. 